\title{
An unification semi-olgorithm for intersection type schemes
}

\author{
Simono Ronchi Delle Rocca \\ Dipartimento di Informatica - Universita' di Torino \\ corso Svizzera 185 - 10149 Torino
}

\section{Introduction.}

The intersection type discipline for $\lambda$-calculus (ITD), defined in [Coppo et al., $1980 \mathrm{~b}$ ], is an extension of the classical functionality theory of Curry [Curry et 81, 1958] .In Curry type discipline type schemes are built from type variables using the constructor $\rightarrow$ (arrow). In the TTD, type schemes are built from type variables and the type constant $\mathrm{b}$ (the universal type) using, as constructars, beside the arrow, the intersection ( $\lambda$ ). The semantics of a type scheme of the shape $\alpha \rightarrow \beta$ is the classical one, the semantics of $a$ type scheme of the shape $\alpha A \beta$ is the intersection of the sets representing the meanings of $\alpha$ and $\beta$, the semantics of $\omega$ is the whole semantic domein. In the ITD every term has ot least one type scheme, and type assignment preserves g-convertibility.

In the Curry type discipline, every term $X$ which can be typed has a principal type scheme (pts), from which all and only the type schemes deducible for $X$ can be derived, by means of substitutions. The problem of computing the pts, if it exists, of a term in the Curry type discipline is decidable, and algorithms to solve it have been proposed by Hindley [Hindley, 1969] (for terms of Combinatory Logic), and by Milner [Milner, 1978] [Milner et 81.,1982] (for terms of $\lambda$-calculus). Milner uses this algorithm in the design of the ML type checker. Both these algorithms are besed on the classical unification algorithm of Robinson [Robinson, 1965].

In the ITD each term $X$, which has a finite set of approximants, has a pts in an extended meening. More precisely, every type scheme deducible for $X$ is derived from its pts by means of a sequence of suitable operations, namely the substitution, the expension and the rise [Ronchi et 81,1984 ].

In this paper the unification problem for intersection type scheme is studied. This problem is semi-decidable. The semi-slgorithm UNIFY solving it is presented, and it is proved that, in the case a solution exists, it finds the more general one. While UNIFY uses also operetions different from substitution, it is conservative with respect to Robinson's unification algorithm. Moreover a semi-aigorithm PP is presented, using UNIFY as essential tool, which, given s term $x$, if $x$ is strongly normalizing computes its pts . Since there is a one-one correspondence between a term and its pts (if it exists), PP con be viewed alsa as a reduction machine, using an innermost reduction strategy. The use of unification between type schemes instead of g-reduction in computing the normal form of a term avoids the necessity of $\alpha$-conversions. 


\section{The intersection type discipline.}

The reader is supposed to have some acquaintance with $\lambda$-colculus; in any case he can refer to [Barendregt,1984], whose notations we will use.

Definition 1. i) The set $T$ of intersection type schemesis inductively defined by:

$\varphi_{j}, \phi_{j}, \ldots \in T$ (i20) (type variables)

$\omega \in T$ (type constant)

$\sigma, \tau \in T \Rightarrow(\sigma \rightarrow \tau) \in T,(\sigma \wedge \tau) \in T$.

ii) A statement is of the form $\sigma x$ with $\sigma \in T$ and $x$ is a variable. $x$ is the subject and 6 the predicate of БM. A hasis scheme is a (possibly infinite) set of statements.

The notion of subtype of a given type scheme is obtained in a straightforward way from Definition 1.i). $\sigma_{1} \rightarrow \sigma_{2} \rightarrow \ldots \rightarrow \sigma_{n} \rightarrow \tau$ is an abbreviation for $\sigma_{1} \rightarrow\left(\sigma_{2} \rightarrow\left(\sigma_{3} \rightarrow \ldots\left(\sigma_{n} \rightarrow \tau\right) \ldots\right)\right)$ and $\sigma_{1} \wedge \sigma_{2} \wedge \ldots \wedge \sigma_{n}$ is on sbbreviation for $\left(\sigma_{1} \wedge\left(\sigma_{2} \wedge \ldots\left(\sigma_{n-1} n \sigma_{n}\right) \ldots\right)\right)$

The simple sementics (for the definition of simple semantics see [Hindley, 1983]) for $T$ can be given in the following way:

Definition 2. Let $/ *\left\langle\mathrm{D}_{-}\right.$, ill $\rangle$be a $\lambda$-model

i) Let $N \in A$; if $\xi$ is a voluetion of veriables in $D$, then $\mathbb{N} N_{\xi}^{\text {th }} \in D$ is the interpretation of $N$ in Mvio $\xi$.

ii) Let $P D=\{X \mid X \subseteq D\}$ and $V:\{\varphi \mid \varphi$ is a type variable $\rightarrow P D$. Then the interpretation of $\sigma \in T$ in $M$ via $V$, notation $\llbracket 6$ I

$\|\omega\|^{\alpha,}=0$

$\| \varphi \rrbracket=V(\varphi)$

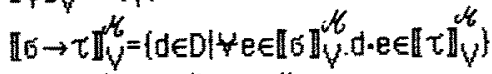

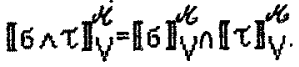

This semantics induces neturally a pre-order relation $\leq$ on $T$, whose intended meaning is : $6 \leq \tau \Leftrightarrow \forall M$. $\|\sigma\|_{V}^{\prime \prime} \llbracket \tau \|_{y}$.

Definition 3. The relotion $\leq$ (and $\sim$ ) on $T$ is inductively defined by:

i) $\tau \leq \tau, \tau \leq \omega, \omega \leq \omega \rightarrow \omega, \tau \leq \tau A \tau, \tau \wedge \sigma \leq \sigma, \tau \wedge \sigma \leq \tau,(\sigma \rightarrow \rho) \wedge(\sigma \rightarrow \tau) \leq \sigma \rightarrow\left(\rho_{A} \tau\right)$,

$\sigma \leq \tau \leq p \Rightarrow \sigma \leq p, \sigma \leq \sigma^{\circ}$ end $\tau \leq \tau^{\prime} \Rightarrow \sigma \wedge \tau \leq \sigma^{\prime} \wedge \tau^{\prime}, \sigma 2 \sigma^{\prime}$ and $\tau \leq \tau^{\prime} \Rightarrow \sigma \rightarrow \tau \leq \sigma^{\prime} \rightarrow \tau^{\prime}$.

ii) $\sigma \sim \tau \Leftrightarrow \sigma \leq \tau \leq \sigma$.

Definition 4. Tyge scheme assignment rules

Let $B$ be a basis scheme, and let $A$ be the set of type free $\lambda$-terms.
(w) B $\mathrm{B}$ X for all $\mathrm{X} \in \mathrm{A}$
(vor) $B U(6 x\}-6 x$
(A) $\frac{B+G X \quad B+\tau X}{B-5 \wedge \tau X}$
(AE) $\frac{B+6 A \tau X}{6 X} \quad \frac{B-6 \wedge \tau X}{\tau X}$ 
$(\rightarrow 1) \frac{B U(\sigma x)^{*}+\tau X}{B-\sigma \rightarrow \tau \lambda X X} \quad(\rightarrow E) \frac{B \vdash \sigma \rightarrow \tau X \quad B \vdash \sigma Y}{B-\tau X Y}$

* where $\sigma x$ is the unique statement of $B$, whose subject is $x$, used to deduce $\tau x$.

(s) $\frac{B+\sigma \times \quad \sigma \leq \tau}{B+\tau X}$

Note that the rule $(A E$ ) is redundant, since it can be directly derived from rule ( $\leq$ ).

Motation. Let $\langle Z, W, z\rangle$ be a triple of either two type schemes and a type variable, or two terms and a variable. $[[z / w]$ denote the type scheme (term) obtained from $z$ by simultaneous replacing each free occurrence of $z$ with $W$. If $z$ ' is a (type) variable, $z\left[z / z^{\prime}\right]$ is an instance of $z$.

Let $\rightarrow_{\beta}$ denote the $\beta$-reducibility, i.e.,

$$
\left.C[(\lambda x . M) N] \rightarrow_{\beta} C[M[x / N]] \quad \text { (where } C l\right] \text { is any context) }
$$

iff $N$ is substitutible for $x$ in $M$ (note that a term $N$ can always become substitutible for a given variable in a term $M$, by means of a renaming of bound variables in M).Let ${ }_{\beta}$ denote $\beta$-convertibility, i.e., the transitive and reflexive closure of $\beta$-reducibility. We recall that a term $X$ is in normal farmiff $A x$. $X{ }_{\beta} x$, and it is in head hormal form iff $x=\lambda x_{1} x_{2} \ldots x_{n} \zeta x_{1} x_{2} \ldots x_{m}$ (n,m 20$)$, where $\zeta$ is any variable and $x_{j} \in \Lambda$ (1<ism). $x$ hes a normel form (a hesd normel form) iff $X \rightarrow{ }^{*}{ }^{X}$ and $X$ is in normal form (head normel form).

The following theorem holds:

Theorem 1 ([Berendregt et al,1981]i) Let $X={ }_{\beta} X^{\prime}$.Then $B \vdash \tau X \Leftrightarrow B \vdash \tau X$.

ii) $\exists B, \tau \neq B . B-\tau X \Leftrightarrow X$ has a head normal form.

iii) $\exists B, \tau . B+\tau X$ and $\omega$ does not occur neither in $B$ nor in $\tau \Leftrightarrow X$ has a normal form.

Without less of generality, we can restrict ourselves to consider only finite bosis schemes.

Definition 5.i) A pair $\langle B, \tau\rangle$, where $B$ is a basis scheme and $\tau$ is a type scheme is switabiefor $X \in \Lambda$, iff there exists a deduction $D$ such that

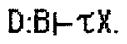

ii) = will denote the synctactical identity between type schemes, basis schemes and psirs.

iii) The equvalence relotion $\sim$ between poirs is so defined:

$\langle B, \tau\rangle \sim\left\langle B^{\prime}, \tau^{\prime}\right\rangle \Leftrightarrow \tau \sim \tau^{\prime}$ and, if, $\forall x$. $\sigma_{j} x(1 \leq i \leq m)$ and $e_{j} x(1 \leq j \leq n)$ are the all and anly statements whose subject is $x$ belonging respectively to $B$ and $B^{\prime}, \sigma_{1} \wedge \ldots \wedge \sigma_{m} \sim \rho_{1} \wedge \ldots \wedge \rho_{n}$.

Now, we will define two aperations on pairs, which preserve suitable pairs, namely the substitution and the expansion.

Definition 6. A substitution $s$ is a finite set of pairs $\left\langle\varphi_{j}, \mu_{j}\right\rangle(1 \leq i \leq n)$, where $\varphi_{j}$ are 
distinct type voriables and $\mu_{j}$ are type schemes. Then, for every pair $\langle B, \tau\rangle$ :

i) $s(\tau)=\tau\left[\varphi_{j} / \mu_{j}\right], s(B)=\{s(\sigma) \times \mid \sigma x \in B)$

ii) $s(\langle B, \tau\rangle)=\langle s(B), s(\tau)\rangle$.

Clearly, if $D: B \vdash-\tau X$ and $s$ is any substitution, $\exists D^{\prime}: s(B)-s(\tau) . D^{\prime}$ is obtained from 0 simply applying s to every basis and type scheme occurring in $D$.

Hotation. Let $L$ be an ordered list. Then $L$ «o will denote the ardered insertion of a into $L$, and $L \gg 8$ will denote the extraction of the maximum element of $L$, whose name is a.

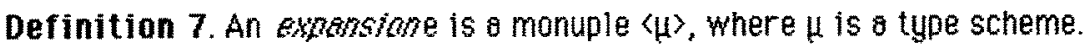

i) Let $L^{e}(B, \tau)$ a list of type scheme,ordered by number of symbols (when two type schemes have the same number of symbols their mutusi order is unimportant). $L^{e}(B, \tau)$ is built in the following woy:

$-L^{E}(B, \tau) \times \mu$

-if $\sigma \in L^{e}(B, \tau)$, and $\delta$ is 8 proper subtype of $\sigma, L^{e}(B, \tau) \ll \delta$.

-for each type scheme $\sigma$, such that $\sigma$ is a subtype of either $\tau$ or o predicate in $B$ :

-if either $\sigma=v \rightarrow \delta$ or $\sigma=v \rightarrow(\delta \wedge, \gamma)$ and $\delta \in L^{e}(B, \tau)$, then $L^{e}(B, \tau) \ll \sigma$.

ii) Let $I=\left\{\varphi_{j} \mid \varphi_{j}\right.$ is a type variable occurring in $\left.L^{e}(B, \tau)\right\}$.

Let $s_{j}=\left\{\left\langle\varphi_{j}, \psi_{i}\right\rangle \mid \varphi_{j} \in l, \psi_{j}\right.$ is a fresh varioble) (1sis2).

iii) $\forall G \in T, e(\sigma)$ is obtained from $\sigma$ by meens of the following procedure:

while $L^{e}(B, \tau) \neq \varepsilon$ ( $\epsilon$ is the empty list) do begin

$L^{e}(B, \tau) \times y$

if $\gamma$ occurs in 6 then replace $\gamma$ in 6 by $s_{1}(\gamma) \wedge s_{2}(\gamma)$

iv) $e(B)=\{e(6) \times \mid 6 \times \in B\}$

end

y)e( $\langle B, \tau\rangle)=\langle\mathrm{e}(\mathrm{B}), \mathrm{e}(\tau)\rangle$.

An expansion $\mathrm{e}$ will be called tata with respect to a type scheme $\sigma$ iff $e(6)=e^{\prime}(\sigma)$, where $e^{\prime}=\langle 6\rangle$.

Let $D \cdot B+\tau X$. Then, if $e$ is an expansion, there exists a deduction $D^{*}:(B)+e(\tau) X$, and $D^{\prime}$ is obtained from D by duplicating some subdeductions of $D$ and by adjoining some opplications of the rule $(\mathrm{Al})$, as can be seen in the following:

Example 1. Let $B=((\varphi \rightarrow \varphi) \rightarrow \alpha y)$. Then $\langle B, \alpha\rangle$ is a suitoble pair for the term $y(\lambda \times x)$. In fact we can show the deduction $D$ :

$$
(\rightarrow E) \frac{B \vdash(\varphi \rightarrow \varphi) \rightarrow \alpha y \quad(\rightarrow 1) \frac{B U\{\varphi x\} \vdash \varphi x}{B \vdash \varphi \rightarrow \varphi \lambda x x}}{B \vdash \alpha y(\lambda x . x) .}
$$

Let $e=\langle\varphi\rangle$. e( $\langle B, \alpha\rangle)=\left\langle\left(\left(\left(\varphi_{1} \rightarrow \varphi_{1}\right) \wedge\left(\varphi_{2} \rightarrow \varphi_{2}\right)\right) \rightarrow \alpha y\right\}, \alpha\right\rangle$ is a suitable pair for $y(\lambda x, x)$. In fact there exists a deduction $D$ : 


\begin{tabular}{|c|c|c|}
\hline $\begin{array}{l}(\rightarrow 1) \\
(A 1)\end{array}$ & $\frac{e(B) \cup\left\{\varphi_{1} x\right)-\varphi_{1} x}{e(B)-\varphi_{1} \rightarrow \varphi_{1} \lambda x . x}(\rightarrow 1)$ & $\frac{e(B) \cup\left(\varphi_{2} x\right)+\varphi_{2} x}{e(B)+\varphi 2^{\prime} \rightarrow \varphi_{2} \lambda x . x}$ \\
\hline$(\mathrm{B}) \mapsto\left(\left(\varphi_{1} \rightarrow \varphi_{1}\right) \wedge\left(\varphi_{2} \rightarrow \varphi_{2}\right)\right) \rightarrow$ & $e(B)+\left(\varphi_{1}-\right.$ & $\left.\rightarrow \varphi_{1}\right) \wedge\left(\varphi_{2} \rightarrow \varphi_{2}\right) \wedge \times . x$ \\
\hline
\end{tabular}

The notion of instance can be naturally extended to substitutions and expansions; we will say that a substitution $s=\left\langle\left\langle\varphi_{j}, \sigma_{j}\right\rangle\right\}$ is an instance of $s^{\prime}=\left\{\left\langle\varphi_{j}, b_{j}{ }_{j}\right\rangle\right\}$ iff $\sigma_{j}$ is an instance of $\sigma^{\prime}$, and an expansion $e=\langle\mu\rangle$ is an instance of $e^{\prime}=\left\langle\mu{ }^{\prime}\right\rangle$ iff $\mu$ is an instence of H.

Definition 8.i) A chain $\mathrm{c}$ is a finite sequence of operations of substitutions and expensions.

ii) Two chains $c_{1}$ and $c_{2}$ are equiglent (notation $c_{1}=c_{2}$ ) iff:

- if $\left\langle\mu_{1}, \ldots,\left\{\mu_{n}\right\rangle\right.$ and $\left\langle\mu_{1}^{\prime}>, \ldots,\left\langle\mu_{m}^{\prime}\right\rangle\right.$ are alland only the intersections occurring respectively in $c_{1}$ and $c_{2}, \mu_{1} \wedge \ldots \wedge \mu_{n}$ is an instance of $\mu_{1}^{\prime} \wedge \ldots \wedge \mu_{m}^{\prime}$.

- if $s_{1}, \ldots, s_{n}$ and $s_{1}{ }_{1}, ., s_{m}^{\prime}$ are all and only the substitutions occurring respectively in $c_{1}$ and $c_{2}, U_{1 \leq i \leq n} s_{j}$ is an instance of $U_{1 \leq i \leq m} s_{j}$. Note that $c_{1}=c_{2}$ does not imply $c_{1}\langle\langle B, \tau\rangle)=c_{2}\langle\langle B, \tau\rangle)$.

Notation. Let $0 p_{j}$ be an operation of expension or substitution (1sisn). We will denote with $O p_{1} . O p_{2} \ldots O p_{n}$ the chain $c$ such that $c(6)=O p_{n}\left(0 p_{n-1}\left(\ldots o p_{1}(6)\right) \ldots\right)$ and with $c_{1} c_{2}$ the chain which is the concetenation of the two chains $c_{1}$ and $c_{2}, i . e, c(6)=c_{2}\left(c_{1}(\sigma)\right)$.

Theorem 2.([Ronchi et al.,1984]).i) Let $c$ be any chain. If $\langle B, \tau\rangle$ is a suiteble pair for $X$, then $c(\langle B, \tau\rangle)$ is also a suitable pair for $\mathrm{x}$.

ii) Let $c$ be a chain, such that $c(\langle B, G\rangle)=\left\langle B^{\prime}, G^{\prime}\right\rangle$, where $G^{\prime}=\mu A \vee$. Then $3 c^{\prime}$ such that: $c^{\prime}=e . c^{\prime \prime}$, where $e=\langle 6\rangle, C^{\prime}(\langle B, 6\rangle)=\left\langle B^{\prime}, 6^{\prime}\right\rangle$ and $C==C^{\prime}$.

It is possible to see the operation of exponsion as operation only on type schemes, not necessery on pairs. The expansion $e=\langle\mu\rangle$, applied on the type scheme $\tau$, is defined as in Definition $7 . i i i)$, with $L^{\mathrm{e}}(B, \tau)$ replaced by $L^{\mathrm{e}}(\Phi, \tau)$, where $\Phi$ is the empty set. In what follows we will use 'expansion' to denote indifferently the two operations, since the meaning will be clear from the context.

\section{The unification semi-algorithm.}

The unification problem for type schemes belonging to $T$ could be stated in the following "syntactic" way:

- Given $6, \tau \in T$, find, if it exists, a chain $c$ (of expansions and substitutions), such that $c(\sigma)=c(\tau)$.

But in this formulation of the problem the porticular role of the universal type scheme $\omega$ is not taken into account. In foct, it is netural to impose that $\omega$ can be unified with any type scheme.Then, we can give a "sementic" version of the problem: 
- Given $5, \tau \in T$, find, if it exists, a chain $c$ such that $c(\sigma) \sim c(\tau)$.

But this formulation is too general, and it exels our aims. So, we will define a new equivalence relation between type schemes:

Definition 9.i) $\mathrm{A} \omega$-type scheme is a type scheme in which only the symbol $\omega$ occurs.

ii) $\approx$ is inductively defined as follows:

(Note that $\alpha \wedge \beta \neq \beta \wedge \alpha)$.

$\alpha, \beta w$-type schemes $\Rightarrow \alpha \approx \beta$

$\alpha=\beta \quad \Rightarrow \alpha \approx \beta$

$\alpha \approx \alpha^{2}, \beta \approx \beta^{2} \quad \Rightarrow \alpha \rightarrow \beta \approx \alpha \rightarrow \alpha^{\prime}, \alpha \wedge \beta \approx \alpha^{*} \wedge \beta^{2}$.

We can now try to give a third formulation of the unification problem:

$-G i v e n$ $6, \tau \in T$, find, if it exists, a chain $c$ such that $c(\sigma) \approx c(\tau)$.

But now the problem has always a solution, the trivial one $c(\sigma) \approx c(\tau) \approx w$. The correct formulation of the unification problem must impose that the trivial solution can be choosen only in the case no other solution exists.

Then the final formulation of the problem is:

$-G i v e n$, $\tau \in T$, find, if it exists, a chain $c$ such that $c(\sigma) \approx c(\tau) \neq 0$.

This problem is semi-decidable. In fact, in the following section it will be possible to see that it is equivolent to the problem:

$-\exists<B, \tau\rangle \cdot B \vdash \tau \times$ ?

which is clearly semi-decidable (by Theorem 1.i).

The semi-algorithm UNIFY we will show solves the problem in the most general way, i.e., it finds the most general unifying chain, if it exists, otherwise it does not stop, as will be proved in Theorem 3 .

\section{Semi-algorithm UNIFY}

UNIFY $(\sigma, \tau)=U(\sigma, \sigma, \tau, \tau)$, where $U\left(\sigma, \sigma^{\circ}, \tau, \tau\right)=\tau^{\circ}$ (if defined), where:

1. if 6 is a type variable then if $\tau=\sigma$ then $s=$ 雨 else

if 6 occurs in $\tau$ then $c^{\prime}=s$, where $s=\{\langle\varphi, \omega\rangle,\langle 6, \omega\rangle \mid \varphi$ occurs in $\tau\}$

else $c^{\circ}=s$, where $s=(\langle\sigma, \tau\rangle)$;

2. if $\sigma=\omega$ then $c^{\prime}=s$, where $s=\{\langle\varphi, \omega\rangle \mid \varphi$ occurs in $\tau\}$;

3. if $b=61 \rightarrow 62$ then

3.1. if $\tau$ is a varioble then

if $\tau$ occurs in 6 then $c=s$, where $s=\{\langle\varphi, \omega\rangle,\langle\tau, \omega\rangle \mid \varphi$ occurs in 6$\}$

else $c^{\circ}=s$, where $s=\{\langle\tau, 6\rangle\}$

3.2. if $\tau=\omega$ then $c^{\prime}=s$, where $s=\{\langle\varphi, \omega\rangle \mid \varphi$ occurs in 6$\}$

3.3. if $\tau=\tau_{1} \rightarrow \tau_{2}$ then

if $c_{1}=U\left(\sigma_{1}, \sigma^{\circ}, \tau_{1}, \tau^{\prime}\right)$ and $c_{2}=U\left(c_{1}\left(\sigma_{2}\right), c_{1}\left(\sigma^{\circ}\right), c_{1}\left(\tau_{2}\right), c_{1}(\tau)\right)$

then $c=c_{1} \cdot c_{2}$

3.4.if $\tau=\tau_{1} \wedge \tau_{2}$ then

Let $e=\left\langle\sigma^{\prime}\right\rangle$, then if $c_{1}=U\left(e\left(\sigma^{\circ}\right), e\left(6^{\prime}\right), e\left(\tau^{2}\right), e\left(\tau^{\prime}\right)\right)$

then $c^{*}=e^{\circ} c_{1}$ 
4. If $\sigma^{=5} 1^{\wedge \sigma_{2}}$ then:
4.1. [ identicol to point 3.1 ]
4.2. [ identicol to point 3.2 ]
4.3. if $\tau=\tau_{1} \rightarrow \tau_{2}$ then
let $e=\langle\tau\rangle$ then if $c_{1}=U\left(e\left(\sigma^{\circ}\right), \varepsilon\left(6^{\circ}\right), \varepsilon\left(\tau^{\prime}\right), \varepsilon\left(\tau^{\prime}\right)\right)$
then $c^{\prime}=e . c_{1}$
4.4. if $\tau=\tau_{1} \wedge \tau_{2}$ then
if $c_{1}=U\left(\sigma_{1}, \sigma^{\prime}, \tau_{1}, \tau^{\prime}\right)$ and if $c_{2}=U\left(c_{1}\left(\sigma_{2}\right), c_{1}\left(\sigma^{\prime}\right), c_{1}\left(\tau_{2}\right), c_{1}\left(\tau^{\prime}\right)\right)$
then $c^{\prime}=c_{1} \cdot c_{2}$.

Exomple 2. Let $\alpha, \beta, \gamma, \delta, \mu, \vee$ be type variables.

i) If $\sigma=\alpha \rightarrow \omega \rightarrow \beta$ and $\tau=(\alpha \rightarrow \delta) \rightarrow(\mu \rightarrow v) \rightarrow \gamma$, UNIFY $(\sigma, \tau)=c-s_{1}, s_{2}, s_{3}$, where:

$s_{1}=\{\langle\alpha, \omega\rangle,\langle\delta, \omega\rangle\}, s_{2}=\{\langle\mu, \omega\rangle,\langle v, \omega\rangle\}, s_{3}=\{\langle\beta, \gamma\rangle)$, and $\left.c(\sigma) \approx c(\tau) \approx \omega \rightarrow \omega\right) \rightarrow \gamma$.

ii) If $\sigma=\alpha \wedge(\alpha \rightarrow \beta)$ and $\tau=(\mu \wedge(\mu \rightarrow v)) \rightarrow v$, UNIFY $(\sigma, \tau)$ does not stop. In fact:

$\operatorname{UNIFY}(\sigma, \tau)=\operatorname{UNIFY}(\mathrm{e}(\sigma), \mathrm{e}(\tau)) \quad($ where $\mathrm{e}=\langle\tau\rangle)=\operatorname{UNIFY}\left(\sigma, \tau^{\prime} \wedge \tau^{\prime \prime}\right) \quad$ (where $\tau^{\prime}$ and $\tau^{\prime \prime}$ are instences of $\tau)=U N I F Y\left(s(\alpha \rightarrow \beta), s\left(\tau^{\prime \prime}\right)\right)$ (where $\left.s=\left\{\left\langle\alpha, \tau^{\prime}\right\rangle\right\}\right)=$

$=$ UNIFY $\left(\tau^{\prime} \rightarrow \beta, \tau^{\prime \prime}\right)=\operatorname{UNIFY}\left(\tau^{\prime} \rightarrow \beta, \sigma^{\circ} \rightarrow v^{\prime}\right)$ (where $\sigma^{*}$ is an instance of $\sigma$ and $v^{\prime}$ is a new type veriable $=$ if $c=U N I F Y\left(\tau^{\prime}, \sigma^{\circ}\right)$ then UNIFY $\left(\alpha(\beta), c\left(v^{\prime}\right)\right)$

and this function is undefined since $\tau$ and $\sigma$, ore instences of $\sigma$ and $\tau$.

To prove that this semi-algoritnm is correct and (in some sense) complete, we need a further definition:

Definition $10 . i$ ) Let $\sigma, \tau \in T$, and let $\sigma^{\prime}$ and $\tau^{\prime}$ be subtypes respectively of $\sigma$ and $\tau$. Two occurrences of $\sigma^{\prime}$ and $\tau^{\prime}$ in $\sigma$ and $\tau$, are correspondingiff:

$-\sigma=\sigma^{\circ}$ and $\tau=\tau^{\circ}$

$-\sigma=\alpha \rightarrow \beta$ and $\tau=\alpha^{\prime} \rightarrow \beta^{\prime}\left(\sigma=\alpha A \beta\right.$ and $\left.\tau=\alpha^{\prime} A \beta^{\prime}\right)$ and the occurrences of $\sigma^{\prime}$ and $\tau$

ore corresponding either in $\alpha$ and $\alpha^{\prime}$ or in $\beta$ and $\beta^{\prime}$.

ii) Let $c=0 p_{1} \ldots O p_{n}$ be o chain such thot $c(\sigma) \approx c(\tau)$. $c$ is o proper cheinunifying $\sigma$ and $\tau$ iff $\forall i(1 \leq i \leq n)$. there exist no two corresponding occurrences of subtypes of $o p_{1} \ldots o p_{i}(\sigma)$ and $\mathrm{op}_{1} \ldots \mathrm{op}_{j}(\tau)$ (soy $\sigma_{j}$ and $\tau_{j}$ ) such thot:

$\sigma_{j}, \tau_{i} \approx \omega$ and $\exists j>i . O p_{1} . O p_{j}\left(\sigma_{j}\right) \approx o p_{j} \ldots o p_{j}\left(\tau_{j}\right) \approx \omega$.

Roughtly speaking, a proper chain unifying two given type schemes is a chain in which a substitution of a type variable with the constant $\omega$ is used only in order to unify two subtypes one of which is $w$.

Then we are sble to prove:

Theorem $3 . i)$ (Correctness) If UNIFY $(\sigma, \tau)=c$, then $c(\sigma) \approx c(\tau)$.

ii) (Completeness) Let $\sigma, \tau \in T$ be such that there exists a proper chain $c$ unifying $\sigma$ and

$\tau$. Then UNIFY $(\sigma, \tau)=\varepsilon^{\prime}$, where $c$ is o proper chain unifying $\sigma$ and $\tau$, and $c=c^{\prime} \cdot c^{\prime \prime}$ for some $c$ "(i.e., $c$ ' is the minima) chain unifying $\sigma$ and $\tau$, in the sense that every other 
some $c^{\prime \prime}\left(i . e, \mathrm{c}^{*}\right.$ is the minimal chain unifying $b$ and $\tau$, in the sense that every other proper chain unifuing $\sigma$ and $\tau$ must contain (an instance of) every operation occurring in $\left.\mathrm{C}^{\prime}\right)$.

Proof. i) Easy, by induction on the lenght of $c$.

ii) By induction on the pair $\langle(c), n(6, \tau)\rangle$ (we assume the lexicographical order between pairs), where:

- I(c) is the lenght of $c$, i.e., the sum of the number of expensions occurring in $c$ and the cordinality of the union of all the substitutions occurring in $c$.

$-n(\sigma, \tau)$ is the total number of sumbols occurring in $\sigma$ and $\tau$.

The cose $I(c)=0$ and $l(c)=1$ are obvious.

Let $1(c)>1$.In the case of is a type variable, we must distinguish two cases, according to $\tau$ contains or not occurrences of 6 . In the first case obviously there is no a proper chain unifying $\sigma$ and $\tau$. Otherwise, UNIFY makes the substitution $s=\{\langle\sigma, \tau\rangle\}$. Obviously this is the minimel between all the proper unifying chains composed only of substitutions ( see IRobinson, 1965). It eesy to see that every proper chein unifying 5 and $\tau$ in which some operations of expansion occur is at least of length 2 (it must contain at least one substitution, since the expansion generates new type variables) and it is always equivalent to a chain composed by a single substitution.

In the case $\sigma \approx \omega$, UNIFY $(\sigma, \tau)=s$, where $s=\{\langle\varphi, \omega\rangle \mid \varphi$ occurs in $\tau\}$. Obviously $s$ is the minimal chain, since every chain unifying $\sigma$ and $\tau$ is such that $c(\sigma) \approx c(\tau) \approx \omega$.

In the case $\sigma=\sigma_{1} \wedge \sigma_{2}$ and $\tau=\tau_{1} \wedge \tau_{2}$, the proof follows directly from the induction hypothesis.

In the case $\sigma_{=}=\sigma_{1} \rightarrow \sigma_{2}$ and $\tau=\tau_{1} \rightarrow \tau_{2}$, if $c$ is such that $c\left(\sigma_{)}\right)=c\left(\sigma_{1}\right) \rightarrow c\left(\sigma_{2}\right)$ and $c(\tau)=c\left(\tau_{1}\right) \rightarrow c\left(\tau_{2}\right)$, the proof follows directly from the induction hypothesis. In the case $c(\sigma) \approx c(\tau) \approx \mu \wedge \rho$, by Theorem 2.ii), there exists $c_{1}=e . c_{2}$, where $e=\langle\sigma \wedge \tau\rangle$, such thot $c_{1}(\sigma) \approx c_{1}(\tau)$ and $c==c_{1}$. So $c_{2}$ is a proper unifying chain for $e(6)$ and $e(\tau)$, and, by

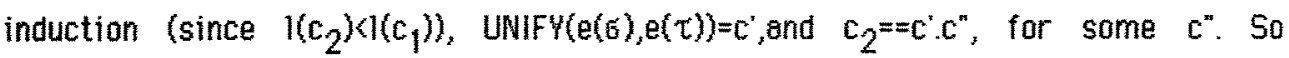
$c_{1}==e . c^{*} \cdot c^{\prime \prime}==c$, and the proof is given, since UNIFY $(\sigma, \tau)=e . c^{2}$.

Consider now the cose $\sigma_{0}=\sigma_{1} \wedge \sigma_{2}$ and $\tau=\tau_{1} \rightarrow \tau_{2}$.

If there exists a proper chain $c$ unifying 6 and $\tau$, c must contain an operation of total expansion with respect to $\tau$. Let $e=\langle\tau\rangle$; by Theorem2.ii) there exists $c_{1}$ such that $c_{1}=e . c_{2}$ and $c_{1}(\sigma) \approx c_{1}(\tau)$ and $c_{1}==c_{\text {. Then }} c_{2}$ is a proper chain unifying $e(\sigma)$ and $e(\tau)$, and $l\left(c_{2}\right)<1(c)$. So, by induction hypothesis, UNIFY $(e(\sigma), e(\tau))=c^{\prime}$, where $c_{2}==c^{\prime} \cdot c^{\prime \prime}$, for some $c^{\prime \prime}$ and $c==c_{1}==e . c^{*} \cdot c^{*}$. Then the proof is given, since UNIFY $(\sigma, \tau)=e . c^{*}$.

Moreover, the semi-algorithm UNIFY is conservative with respect to Robinson's unification algorithm R. More precisely:

Property 1. Let $6, \tau$ be type schemes without occurrences of the symbols $A$ and $\omega$. If $R(\sigma, \tau)=s$, where $s$ is some substitution, then UNIFV $(\sigma, \tau)==s$; if $R(\sigma, \tau)$ fails, then UNIFY $(\sigma, \tau)==s$, where $s$ contains only pairs of the shape $\left\langle\varphi_{j}, \omega\right\rangle$, so $s(\sigma) \approx s(\tau) \approx u$. 
Proof. Easy.

\section{Principal poirs.}

Let us introduce the notion of princinol poir, as defined in Ronchi et. al,1984]. First of all, the notion of approximant of a term must be introduced.

Definition 11.i) The set $N$ of anorasimate normal farms is defined from the set of variables plus a new constant symbol $\Omega$ in the following way:

- $\Omega \in N$, $x \in N$ for all variable $x$

- if $x$ is o voriable and $A \in N(A \neq \Omega)$, then $\lambda x . A \in N$

- if $x$ is a variable and $A_{1}, \ldots, A_{p} \in M(p 20)$, then $x A_{1} \ldots A_{p} \in N$

ii) Let $X$ be a term and $A \in N A$ is an aproximent of $X(A \subseteq X)$ iff $\exists X=X_{B}$ such that $A$ metches $X^{*}$ except ot occurrences of $\Omega$ in $A$.

iii) $x(X)=\{A \mid A \subseteq X\}$.

iv) the type assignment rules of Definition 4 are generalized to elements of $N$ simply by adjoining the following rule:

(w') B-WA for all AEN.

The following theorem holds:

Theorem 4.[Ronchi et al.,1984]. $\langle B, \tau\rangle$ is a suitable pair for $M \in \Lambda$ iff $\langle B, \tau\rangle$ is a suitable pair for same $A \in A(M)$

We can define, for an approximate narmal form A, a unique princizal nair (pp(A)) (modula the relotion $\sim$ ) as follows:

Definition 12. Let $A \in N$

i) if $A=\Omega$, then $p p(A) \sim\langle\Phi, \omega\rangle$ ( $\Phi$ is the empty set)

ii) if $A=x$, then $p p(A) \sim\langle(\varphi x), \varphi\rangle$, where $\varphi$ is a type variable

iii) if $A=\lambda \times A^{\prime}$, and $p p\left(A^{\prime}\right)-\left\langle B^{\prime}, \pi^{\prime}\right\rangle$, then:

1) if $x$ occurs in $A^{\prime}, p p(A) \sim\left\langle B^{\prime}-\{6 x\}, 6 \rightarrow \pi^{\prime}\right\rangle$, where 6 is the inter section of the predicates of $B^{\prime}$ whose subject is $X$

2) otherwise, $p p(A) \sim\left\langle B^{\prime}, \omega \rightarrow \pi^{\prime}\right\rangle$

1V) if $A=x A_{1} \ldots A_{n}$ and $p p\left(A_{i}\right) \sim\left\langle B_{j}, \pi_{j}\right\rangle$ (isisn) (we choose a trivial yariant of them such that they are pairwise disjoint), then $p \rho(A) \sim\left\langle U_{1 \leq i \leq n} B_{j} U\left(\pi_{1} \rightarrow \ldots \rightarrow \pi_{n} \rightarrow \varphi t, \varphi\right\rangle\right.$, where $\varphi$ is a type variable which does not occurs in $B_{j}, \pi_{j}(1 \leq i \leq n)$.

The components of $\mathrm{pp}(\mathrm{A})$ are called respectively the princinal hasis scheme and the princinel type scheme of A.

Note that the principal peir is defined modulo names of type variablas.

The principal pair of an approximate normal form $A$ has the property that if $\langle B, G\rangle$ is suitable for $A$, then there exists a chain $c$ of operations of substitution, expansion and rise, such that $\langle B, 6\rangle \sim c(p p(A))$, where the operation of rise is defined as follows:

Definition 13.A riser is a pair of peirs $\left\langle\left\langle B_{1}, B_{2}\right\rangle,\left\langle\varphi_{1}, \ell_{2}\right\rangle\right\rangle$, where $\varphi_{1} \leq \varphi_{2}$ and $B_{2}$ is such 
that, for every $6 x \in B_{1}$, there exists $6^{\prime} x \in B_{2}$ with $5^{\circ} \leq 6$. Then:

i) $r(\tau)=$ if $\tau \sim \rho_{1}$ then $\rho_{2}$ else $\tau$

$r(B)=$ if $\forall \times . \sigma_{j} x \in B(1 \leq i \leq n)$ and $\tau_{j} x \in B_{1}(1 \leq j \leq m) \Rightarrow \sigma_{1} \wedge \ldots \wedge \sigma_{n} \sim \tau_{1} \wedge \ldots \tau_{m}$, then

$\mathrm{B}_{2}$ else $\mathrm{B}$

ii) $r(\langle B, \tau\rangle)=\langle r(B), r(\tau)\rangle$.

In [Ronchi et al., 1984] it is proved thet the operation of rise preserves suitable pairs: namely, if $\langle B, \tau\rangle$ is such thot there exists $D: B-\tau X$, and $r$ is any rise, there exists $\theta$ deduction $D^{\prime}: r(B) 1 r(\tau)$, and $O^{\prime}$ is obtained from $D$ by adjoining to $D$ some applications of rule (s)

Let $\Pi(X)=\{\langle B, \pi\rangle|\exists A \in A(X)<B, \pi\rangle \sim p p(A)\}$, and $F\{\langle B, \pi\rangle \mid \exists A \in N\langle B, \pi\rangle \sim p p(A)\}$.

on Fis possible to define the following preorder relation:

$\langle\mathrm{B}, \pi\rangle \bar{E}_{\omega}\left\langle\mathrm{E}^{\prime}, \pi^{*}\right\rangle \Leftrightarrow \exists \varphi_{1} \ldots \varphi_{n} .\langle\mathrm{B}, \pi\rangle=\left\langle\mathrm{B}^{2}\left[\varphi_{1} / \omega, \ldots, \varphi_{n} / \omega\right], \pi^{2}\left[\varphi_{1} / \omega, \ldots, \varphi_{n} / \omega\right]\right.$.

Property 2. $F_{,} c_{\omega}$ is a meet semilattice isomorphic to $N$.

Then $\Pi(X)$ is an idesl in $F$ and therefore if $\Pi(X)$ is finite there exists a pair $\langle B, \pi\rangle=\sqcup \pi(X)$, where $\langle B, \pi\rangle \in F$ : then $\langle B, \pi\rangle$ is the $p p$ of $X$. otherwise, $U \pi(X)$ does not exist in $F$, and then $X$ hes an infinite set of pp's, as shown in the following:

Theorem $5 . i),(X)$ is finite $\langle B, \pi\rangle=U \pi(X)$ is such that, if $\left\langle B^{*}, \tau\right\rangle$ is suitable for $X$, then there exists a chain $c$ such that $\left\langle B^{*}, \tau\right\rangle \sim c(\langle B, \pi\rangle)$.

ii) $A(X)$ is infinite. For every $\left\langle B^{\prime}, \tau\right\rangle$ suitable for $X$ there exists $\langle B, \pi\rangle \in \Pi(X)$ such that $\left\langle B^{\circ}, \tau\right\rangle \sim C(\langle B, \pi\rangle)$, for some chain $C$.

In order to compute the principal pair of o term $X$, if it exists, the semi-algorithm PP will be shown. PP uses the unification semi-algorithm UNIFY, defined in the preceding section. In this semi-algorithm, the operation $\nabla$ between besis schemes, with at least one statement on every subject, is used. $\nabla$ is so defined:

$B \nabla B^{\prime}=\left(\sigma \wedge \sigma^{\prime} x \mid \sigma x \in B\right.$ and $\left.\sigma^{\prime} x \in B^{\prime}\right) \cup(5 x)\left(\sigma x \in B\right.$ and $B^{\prime \prime}$ has no a statement on $\left.x\right)$ or $\left(\sigma x \in B^{\prime}\right.$ and $B$ has no a statement on $x$ )).

\section{Semi-algorithm PP.}

$P P(X)=\langle B, \pi\rangle$ (if defined), where:

1) if $X$ is a variable then $\langle B, \pi\rangle=\langle(\varphi)\}, \varphi\rangle$ where $\varphi$ is a fresh type variable.

2) if $X=\lambda x . X^{*}$ then

if $P P\left(X^{\circ}\right)=\left\langle B^{\prime}, \pi^{\prime}\right\rangle$ then

if $B^{2}$ contoins $a$ premise on $x$, let $\sigma x$, then $\langle B, \pi\rangle=\left\langle B^{\prime}-\{6 x\}, 6 \rightarrow \pi^{2}\right\rangle$ else

$-\left\langle B^{*}, \omega \rightarrow m^{*}\right\rangle$.

3) if $X=x_{1} x_{2}$ then

if $P P\left(X_{1}\right)=\left\langle B_{1}, \pi_{1}\right\rangle$ and $P P\left(X_{2}\right)=\left\langle B_{2}, \pi_{2}\right\rangle$ then

if UNIFY $\left(\pi_{1}, \pi_{2} \rightarrow \varphi\right)=c$ ( $\varphi$ is a fresh voriable) then

$\langle\mathrm{B}, \pi\rangle=\left\langle c\left(\mathrm{~B}_{1}\right) \nabla c\left(\mathrm{~B}_{2}\right), c(\varphi)\right\rangle$. 
Remember that a term $X$ is colled sirangly normolizing iff $X$, and every its subterm, possess a normal form.

Thearem 6.PP $(X)=\langle B, \pi\rangle \Leftrightarrow X$ is strongly normalizing and $p p(X) \sim\langle B, \pi\rangle$.

The proof will be given in the following section.

It is possible to define a set of unification algorithms UNIFY $(i 20)$, each one unifying with $\omega$, at every step, all the subtypes occurring at depth $2 i$, where the depth of an occurrence of a subtype in a type scheme is defined as follows.

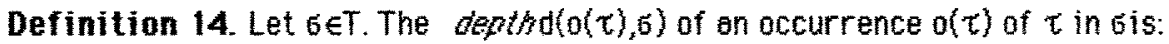

i) if $\tau$ does not occurs in $\sigma$ then $d(\sigma(\tau), \sigma)$ is undefined

ii) if $\sigma=\tau$ then $d(\sigma(\tau), \sigma)=0$

iii) if either $6=\sigma_{1} \rightarrow \sigma_{2}$ or $6=\sigma_{1} A \sigma_{2}$ then

if $d\left(0(\tau), \sigma_{1}\right)=i$ then $d(0(\tau), 6)=i+1$

if $d\left(0(\tau), \sigma_{2}\right)=i$ then $d(0(\tau), 6)=i+1$.

\section{Algorithms UNIFY $\mathbf{y}_{\mathbf{j}}$}

UNIF $Y_{j}(\sigma, \tau)=U_{j}(\sigma, \sigma, \tau, \tau, 0)$ where

$U_{j}\left(\sigma^{\prime}, \sigma^{\prime}, \tau, \tau^{\prime}, j\right)=c$ where

if $j 2 i$ then $c=U\left(\omega, \sigma^{\circ}, \omega, \tau^{\prime}\right)$ else

1. If either $\sigma$ or $\tau$ are either a type variable or $\omega$ then $c=U\left(\sigma, \sigma^{\circ}, \tau, \tau^{\prime}\right)$

2. if $\mathrm{b}=\mathrm{\sigma}_{1} \rightarrow \mathrm{\sigma}_{2}$ then

2.1. if $\tau=\tau_{1} \rightarrow \tau_{2}$ then let $c_{1}=U_{i-1}\left(\sigma, \sigma^{\prime}, \tau_{1}, \tau^{2}, j+1\right)$ and

$$
c_{2}=U_{1-1}\left(c_{1}\left(\sigma_{2}\right), c_{1}\left(\sigma^{\prime}\right), c_{1}\left(\tau_{2}\right), c_{1}\left(\tau^{\prime}\right), j+1\right) \text { then } c=c_{1}, c_{2}
$$

2.2. if $\tau=\tau_{1} \wedge \tau_{2}$ then

let $\mathrm{e}=\langle\sigma\rangle$, then let $c_{1}=\mathrm{U}_{\mathrm{i}}\left(\mathrm{e}\left(\sigma^{\prime}\right), \mathrm{e}\left(\sigma^{\prime}\right), \mathrm{e}\left(\tau^{*}\right), \mathrm{e}\left(\tau^{*}\right), 0\right)$

then $c=e_{1}$

3. if $6=\sigma_{1} A \sigma_{2}$ then

3.1. if $\tau=\tau_{1} \rightarrow \tau_{2}$ then

let $\mathrm{e}=\langle\tau\rangle$, then let $c_{1}=\mathrm{U}_{i}\left(\mathrm{e}\left(\sigma^{\circ}\right), \mathrm{e}\left(\sigma^{\circ}\right), \mathrm{e}\left(\tau^{*}\right), \mathrm{e}\left(\tau^{*}\right), 0\right)$

then $c=e . c_{1}$

3.2. if $\tau=\tau_{1} \wedge \tau_{2}$ then

let $c_{1}=u_{j-1}\left(\sigma_{1}, \sigma^{\prime}, \tau_{1}, \tau_{j}+1\right)$ and $c_{2}=u_{j-1}\left(c_{1}\left(\sigma_{2}\right), c_{1}\left(\sigma^{\prime}\right), c_{1}\left(\tau_{2}\right), c_{1}(\tau), j+1\right)$

then $\mathrm{c}=\mathrm{c}_{1} \cdot \mathrm{c}_{2}$.

Let $P_{j}$ be the elgorithm obtained from PP by replacing UNIFY with UNIFY $j$ (i20). The following theorem holds: 
Theorem 7.1) $P P_{i}(X)=\langle B, \pi\rangle \Rightarrow\langle B, \pi\rangle \in \Pi(X)$.

ii) $\langle B, \pi\rangle \in \Pi(X) \Rightarrow \exists i . P P_{i}(X)=\left\langle B_{j}, \pi_{j}\right\rangle$ and $\langle B, \pi\rangle c_{6}\left\langle B_{i}, \pi_{i}\right\rangle$

Proof. Immediate from Theorem 5 and from the definition of the spproximants of a term.

\section{Proof of Theorem 6.}

$\Leftrightarrow \Leftrightarrow$ By induction on the structure of $x$.

For $X$ variable, obvious. For $X=\lambda x X^{\prime}$ or $X=Y Z$, where $Y$ does not reduce to $\lambda x . Y^{\prime}$, for some $Y^{2}$, the proof follows directly from the induction hypothesis.

For $X=(\lambda x . Y) Z, \quad P P(X) \quad=\langle B, \pi\rangle \Rightarrow P P(\lambda x . Y)=\left\langle B_{1}, 6 \rightarrow \tau\right\rangle \quad$ end $P P(Z\rangle=\left\langle B_{2}, \pi_{2}\right\rangle$ and $\langle B, \pi\rangle=\left\langle c\left(B_{1}\right) \nabla c\left(B_{2}\right), c(\varphi)\right\rangle$, where $c=U N \mid F Y\left(6 \rightarrow \tau, \pi \pi_{2} \rightarrow \varphi\right)$. It essy too see, by examining the semi-algorithm PP, that, if $\rho \sim \sigma \rightarrow \tau$ and $\delta \sim \pi{ }_{2} \rightarrow \varphi$ and UNIFV $(\rho, \delta)=c^{\prime}$, $\langle B, \pi\rangle \sim\left\langle c^{\prime}\left(B_{1}\right) \cup c^{\prime}\left(B_{2}\right), c^{\prime}(\varphi)\right\rangle$. Then the proof is given by induction on the normal forms of $\lambda x . Y$ and $Z$, which exist since $X$ is strongly normalizing by hypothesis.

$\Leftrightarrow)$ Let $B$ be a basis scheme, $\alpha$ be a type scheme $\neq \omega$, and $M$ be a $\lambda-$ term.

Let us define the predicate:

$P(B, x, M) \Leftrightarrow P P(M)$ is defined and $\exists c<B, 0>\cdots C(P P(M))$ and $M$ is strongly normalizing.

Let $x \vec{M}$ denote $x M_{1} \ldots M_{n}$, for $n \geq 0$, and let $F V(M)$ denote the set of variables occurring free in $M$.

Property $3, j) P(B, \alpha \rightarrow \beta, x M)$ and $P(B ;, \alpha, M) \Rightarrow P(B \cup B ;, \beta, x M N)$.

ii) $P(B U f \alpha x), \beta, M x)$ and $x \notin F V(M)$ and $B$ does not contain premises on $x \Rightarrow$ $P(B, \alpha \rightarrow \beta, M)$.

iii) $P\left(B, \sigma_{1}, A \sigma_{2}, M\right) \Rightarrow P(B, 6, M)$ and $P\left(B, \sigma_{2}, M\right)$.

iv) $P(B, \sigma, M)$ and $s \leq \tau \Rightarrow P(B, \tau, M)$.

Proof. i) Let $x \vec{M}=x M_{1} \ldots M_{m} \quad P\left(B^{*}, \alpha, N\right) \Rightarrow P P(N)=\left\langle E^{*}, \pi^{\prime}\right\rangle$ and $\exists c .\left\langle B^{*}, \alpha\right\rangle \sim c(P P(N))$. $P(B, \alpha \rightarrow B, x \vec{M}) \Rightarrow P P(x \vec{M})=\left\langle\left(\pi_{1} \rightarrow \ldots \rightarrow \pi_{m} \rightarrow \varphi x\left|\nabla B, \nabla \ldots \nabla B_{m}, \varphi\right\rangle\right.\right.$, where $\varphi$ is a fresh variable and $P P\left(M_{j}\right)=\left\langle B_{i}, \pi_{i}\right\rangle$, and $\exists c^{\prime}\langle B, \alpha \rightarrow B\rangle \sim C^{*}(P P(x \vec{M}))$. So UNIFY $\left(\varphi, \pi^{*} \rightarrow \varphi\right)=5$, where $S=\left\{\left\langle\varphi, \pi^{2} \rightarrow \phi\right\rangle\right\}$ (申 is fresh), which implies $P P(x \overrightarrow{M N})=\left\langle\left\{\pi_{1} \rightarrow \ldots \rightarrow \pi_{m} \rightarrow \pi^{\prime \prime} \rightarrow \phi x\right\} \nabla B_{1} \nabla \ldots \nabla B_{m} \nabla E^{\prime}, \phi\right\rangle$. Let $c^{\prime \prime}=c . c^{\prime} . s^{\prime}$, where $s^{\prime}=\{\langle\phi, \beta\rangle\}$. $\left\langle B \cup B^{\prime}, \beta\right\rangle \sim C^{\prime \prime}(P P(x \vec{M} N))$, since $P P(N)$ and $P P(x \vec{M})$ ore disjoint, then $P\left(B \cup B^{*}, \beta, x \overrightarrow{M N}\right)$.

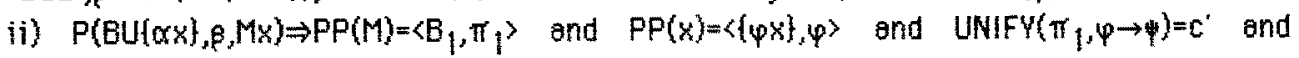
$P P(M x)=\left\langle c^{\prime}\left(B_{1}\right) \nabla\left(c^{\prime}(\varphi) x\right), c^{\prime}(\phi)\right\rangle$ where $\phi$ is fresh and $B_{1}$ does not contain premises on $x_{2}$ and moreover $\exists c .<6 U\left\{(\alpha x), \beta>\sim c\left(\left\langle c^{\prime}\left(B_{1}\right) \nabla(c)(\varphi) x\right), c(\varphi)\right\rangle\right.$. Note that $c^{\prime}$ does not contain any expansion involving the type varisble $\psi$.

Then $\langle B, \alpha \rightarrow \beta\rangle=c\left(\left\langle c^{\prime}\left(B_{1}\right), c^{\prime}(\varphi) \rightarrow c^{\prime}(\varphi)\right\rangle\right.$ ) (since $B_{1}$ does not contoin premises on 
$x)=c\left(\left\langle c^{\prime}\left(B_{1}\right), c^{\prime}\left(\pi_{1}\right)\right\rangle\right)=c^{\prime} c\left(\left\langle B_{1}, \pi_{1}\right\rangle\right)$, and then $P(B, \alpha \rightarrow \beta, M)$

iii) and iv) are immediate.

Then define, by induction on the structure of type schemes $\$$ wi, the following computability predicate:

$\operatorname{Comp}(B, \varphi, M) \Leftrightarrow P(B, \varphi, M)$

$\operatorname{Comp}(B, \sigma \rightarrow \tau, M) \Leftrightarrow\left(\operatorname{Comp}\left(B^{*}, \sigma, N\right) \Rightarrow \operatorname{Comp}\left(B \cup B^{*}, \tau, M N\right)\right.$

$\operatorname{Comp}\left(B, \sigma_{1}{ }^{A} \sigma_{2}, M\right) \Leftrightarrow \operatorname{Comp}\left(B, \sigma_{1}, M\right)$ and $\operatorname{Comp}\left(B, \sigma_{2}, M\right)$

$H$ is easy to prove, by induction on the structure of $M$, that Comp is invariant under $\beta$-convertibility.

Lemme 1. i) $\mathrm{P}(\mathrm{B}, 6, \mathrm{xM}) \Rightarrow \operatorname{Comp}(B, 6, x \vec{M})$.

ii) $\operatorname{Comp}(B, 6, M) \Rightarrow P(B, 5, M)$.

Proof.i) and ii) by simultaneous induction on $\sigma$.

$\sigma$ is a type variable. i) and ii) follow from the definition of Comp.

$6=\alpha \rightarrow \beta$.

i) $\operatorname{Comp}\left(B^{\prime}, \alpha, N\right) \Rightarrow P\left(B^{\prime}, \alpha, N\right)$ (by induction hypothesis).

$P(B, \alpha \rightarrow \beta, x M)$ and $P\left(B^{*}, \alpha, N\right) \Rightarrow P\left(B \cup B^{*}, \beta, x \overrightarrow{M N}\right)$ (by Property 3$) \Rightarrow C \operatorname{Comp}\left(B \cup B^{\prime}, \beta, x \vec{M} N\right)$ (by induction hypothesis). Then $\operatorname{Comp}\left(B_{3}^{\circ}, \alpha, N\right)$ and $\operatorname{Comp}\left(B \cup B^{\prime}, \beta, \times \vec{M} N\right) \Rightarrow \operatorname{Comp}(B, \alpha \rightarrow \beta, x \vec{M})(b y$ def. of Comp).

ii) Let $x \in F V(M)$ and let $(\alpha x) \in B^{\prime} . P\left(B^{\prime}, \alpha, x\right) \Rightarrow$ Comp $\left(B^{\prime}, \alpha, x\right)$ (by induction).

Comp $(B, \alpha \rightarrow \beta, M)$ and $\operatorname{Comp}\left(B^{\prime}, \alpha, x\right) \Rightarrow \operatorname{Comp}\left(B \cup B^{\prime}, \beta, M x\right)$ (by definition) $\Rightarrow P\left(B \cup B^{\prime}, \beta, M x\right)$ (bu induction $) \Rightarrow P(B, \alpha \rightarrow \beta, M)$ (by Property 3$)$.

$5=\sigma_{1}{ }^{n \sigma_{2}}$

ii) by definition of Comp and by induction hypothesis.

i) $P\left(B, \sigma_{1} \wedge \sigma_{2}, x \vec{M}\right) \Rightarrow P\left(B, \sigma_{1}, x \vec{M}\right)$ and $P\left(B, \sigma_{2}, x \vec{M}\right)$ (by Property 3$) \Rightarrow$ Comp $\left(B, \sigma_{1}, x M\right)$ and Comp $\left(B, \sigma_{2}, x \vec{M}\right)$ (by induction) $\Rightarrow \operatorname{Comp}\left(B, \sigma_{1} \wedge \sigma_{2}, x \vec{M}\right)$ (by definition).

Lemmo 2. Let $\left(x_{1}, \ldots, x_{m}\right) \supset F V(M)$, and let $B$ be such that $\left(\sigma_{j} x_{i}\right) \in B(1 \leq i \leq m) \operatorname{Comp}\left(B^{*}, \sigma_{j}, N_{j}\right)$ $(1 \leq i \leq m)$ and $P P(M)=\langle\underline{B}, \pi\rangle$ and $\exists C .\langle B, \tau\rangle \sim c(\langle\underline{B}, \pi\rangle) \Rightarrow \operatorname{Comp}\left(B \cup B^{*}, \tau, M\left[x_{j} / N_{j}\right]\right)$.

Proof $B y$ induction on $M$. The only not trivial cose is $M=\lambda \times M$.

Then $P P(M)=\langle\underline{B}, \alpha \rightarrow \beta\rangle \Rightarrow P P(M) \sim\langle\underline{B} U(\alpha x), \beta\rangle$, where $\underline{\underline{B}}$ does not contain premises on $x$. $P P(M) \sim\langle\underline{B} U(\alpha x), \beta\rangle$ and $\exists c .\langle B \cup\{\sigma x), \tau\rangle \sim \mathcal{C}(\langle\underline{B} U(\alpha x), \beta\rangle)$ and $\operatorname{Comp}\left(B^{\prime \prime}, \sigma_{, N}, N\right)$ and $\operatorname{Comp}\left(B^{\prime}, \sigma_{j}, N i\right)$ $(1 \leq i \leq m) \Rightarrow$ Comp(BU(6x)UB'UB", $\tau, M\left[x / N, x_{i} / N_{i}\right) \quad(1 \leq i \leq m)$ (by induction) $\Rightarrow$

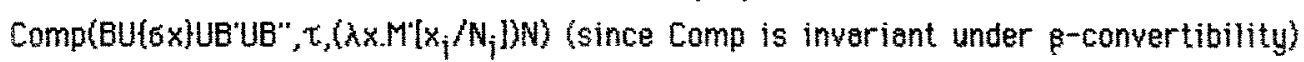
$\Rightarrow$ Comp(BUB', $\sigma \rightarrow \tau, \lambda \times M^{\prime}\left[x_{i} / N_{i}\right]$ ) (by definition).

Then let $P P(M)$ be defined, and let $\left(x_{1}, \ldots, x_{m}\right\}=F V(M)$, and let $\langle B, \tau\rangle_{\infty}(P P(M))$ and let $\left\{\sigma_{i} x\right\} \in B(1 \leq i \leq m)$. Then Comp $\left(B, \sigma_{j}, x_{j}\right)$. By Lemma 2 , this implies Comp(B, $\left.\tau, M\right)$, which implies $P(B, \tau, M)$, by Lemma 1.ii). 


\section{The intersection type discipline without 0.}

In ICappo et al.,1980 al, for the first time an intersection type discipline was introduced, built from a set of type variables, without any constant. More precisely, the type schemes are defined os in Definition 1 , without the constant $\omega$, and the assignment rules are as in Definition 4 , without the rule (w). The definition of pairs, equivalence relation $\cdots$, and operetions of pairs remoin unchenged. It is passible to define o principal poir in this discipline, in the following way:

Definition 15. Let $X$ be a normal form. $p p^{\prime}(X)$ is so defined:

i)if $x=x$ then $p p^{\prime}(x)<\{(\varphi x), \varphi\rangle$ where $\varphi$ is a fresh type warioble

ii) if $x=\lambda \times X^{\prime \prime}$ and $p p^{\prime}\left(X^{\prime \prime}\right) \sim\left\langle B^{\prime}, \pi^{\prime \prime}\right\rangle$, then:

1) if $x$ occurs in $x^{\prime}$ then $p p^{\prime}(x) \sim\left\langle B^{\prime}-\{6 x\}, 6 \rightarrow \pi\right\rangle$, where 6 is the

intersection of the predicates in $B^{\prime}$ whase subject is $x$

2) otherwise $p p^{\prime}(x) \sim\left\langle B^{\prime}, \varphi \rightarrow \pi^{\prime 2}\right\rangle$, where $\varphi$ is fresh.

iii) if $X=x X_{1} \ldots X_{n}$ and $p p^{2}\left(X_{j}\right) \sim\left\langle B_{j}, \pi_{i}\right\rangle$ then $p p^{\prime}(X) \sim\left\langle U_{1 \leq i \leq n} B_{i} U\left\{\pi_{1} \rightarrow \ldots \rightarrow \pi_{n} \rightarrow \varphi X\right), \varphi\right\rangle$, where $\varphi$ is fresh.

The proof that $p p^{\prime}(X)$ is really the principol poir of $X$, in the sense thet oll ond oniy the type scheme deducible for $X$ are obtained from $p p^{\prime}(X)$ by means of chains of substitutions, expansions and rise, is a particular case of the proof that, for $A \in N$, $p p(A)$ is the principel poir of $A$, given in (Roncht et ai., 1984). Moreover an algortthm PP' can be define, which differs from PP only in the point 2), which must be replaced by:

2) if $X=\lambda \times X^{\prime}$ then

if $\mathrm{PP}^{\prime}\left(X^{\prime}\right)=\left\langle\mathrm{B}^{\circ}, \pi^{\prime}\right\rangle$ then

if $B^{\prime}$ contains premise on $X$, let $6 x$, then $P^{\prime}(X)=\left\langle B^{\prime}-(6 x), 6 \rightarrow \pi^{\prime}\right\rangle$

else $P P^{\prime}(X)=\left\langle B^{\prime}, \varphi \rightarrow \pi^{\prime}\right\rangle$, where $\varphi$ is o fresh type vorioble.

Then we obtoin, as corollary of Theorem 6 , the following:

Theorem 8 . In the intersection type discipline without the constant $\omega$, there exists 8 peir suitable for $X$ iff $X$ is strongly normolizing.

This result is stated, but not proved, in [Coppo et $1 ., 1980$ a].

Aknoledgments. The author is very grateful to Paolo Busso and Mauro Berta, who gave an essential contribution in designing and implementing the semi-sigorithms UNIFY and PP. 


\section{Reterences.}

[Barendregt,1984] Barendregt $H$., The Lambda Colculus: its syntox and sementics, North Hollend, (Amsterdem).

[Berendregt et el.,1981] Berendregt $H$, Coppo M.,Dezeni $M$., A filter $\lambda$-model and the completeness of type ossignment, Journal of Symbolic Logic, 64, 4 .

[Coppo et 81.,1980 8] Coppo M., Dezoni M., An extension of the basic Functionality Theory for the L-calculus, Notre Dame Journal of Formal Logic, 21,4.

[Coppo et al,1980 b] Coppo M., Dezani M., Venneri B., Principal type scheme and $\lambda$-calculus semantics, in: J.P.Seldin,J.R.Hindley eds, To H.B.Curry.Esseys on Combinatory Logic, $\lambda$-celculus and Formalism, Acedemic Press, London, 1960,pp 535-560.

[Curry et $81 ., 1958$ ] Curry H.B. Feys R., Combinatory Logic, vol.1, Nart Holland (Amsterdem).

[Hindley.1969] Hindley R.,The principal type scheme 8s an object in combinatory logic, Trans. Amer. Math. Soc., 146.

[Hindley, 1983) Hindley R. The completeness theorem for typing $\lambda$-terms, Theoretical Computer Science, 22.

[Milner, 1978] Milner R., A theory of type polimorphism in programming, J. Comput. System $5 \mathrm{ci}, 17$.

[Milner et al.,1982] Milner R., Damos L.,Principol type schemes for functional programs, 9 -th symp. on Principle of programming languages.

[Robinson, 1965] Robinson J.A., A mschine oriented logic bosed on the resolution principle, Journal of ACM, 12.

[Ronchi et 81.,1984] Ronchi Dollo Rocco S., Venneri B., Principal type scheme for en extended type theory. Theoretical Computer science, 28 . 